\title{
Un estudio exploratorio acerca de las fortalezas del carácter en niños argentinos
}

\author{
fn Exploratory Study fbout Character Strengths in frgentinean Children \\ Um estudo exploratório dos pontos fortes do caráter em crianças argentinas
}

\author{
Aldana Sol Grinhauz, Alejandro Castro Solano* \\ Universidad de Buenos Aires
}

Doi: dx.doi.org/10.12804/apl33.01.2015.04

\section{Resumen}

Esta investigación se centró en el segundo pilar de la Psicología Positiva - las virtudes y fortalezas del carácter- con el fin de explorar mediante un enfoque mixto, es decir, émico-ético, las fortalezas autopercibidas en 163 niños, $51 \%$ varones y $49 \%$ mujeres pertenecientes a la Ciudad de Buenos Aires (Argentina). El $40 \%$ de los participantes tenía diez años, el $28 \%$ tenía once años y el $32 \%$ tenía doce años. Se les solicitó a los participantes que describieran con sus palabras las fortalezas del carácter que reconocían en sí mismos.

Dentro de las fortalezas más reportadas por los participantes se encontraron la integridad y la bondad. Dentro de las menos reportadas como presentes, se hallaron la espiritualidad, el amor por el conocimiento y la prudencia. Asimismo, se encontraron diferencias en las fortalezas autopercibidas de acuerdo con el sexo y la edad. En las respuestas brindadas por los niños no surgieron fortalezas distintas a las previstas en la clasificación Values in Action.
Palabras clave: Psicología Positiva, fortalezas, niños, émico

\section{flbstract}

This study focused on the second pillar of positive psychology -virtues and character strengths- with the purpose of exploring by a mixed approach - that is, emic - etic- self-perceived strengths in 163 children, 51\% male and $49 \%$ female from Buenos Aires City (Argentina). Forty percent of the participants were 10 years old, $28 \%$ were 11 years old and $32 \%$ were 12 years old. Participants were asked to describe with their own words the character strengths they recognized in themselves. Integrity and goodness were the most frequently reported strengths, while spirituality, love for knowledge and prudence were the least mentioned strengths. Sex and age differences were found in relation to self-perceived strengths. No strengths different from those included in the Values in Action classification arised from the results.

* Aldana Sol Grinhauz, Facultad de Psicología, Universidad de Buenos Aires; Alejandro Castro Solano, Facultad de Psicología, Universidad de Buenos Aires.

La correspondencia relacionada con este artículo debe ser dirigida a Aldana Sol Grinhauz O’Higgins 2613 - 1. ${ }^{\circ}$ A, Buenos Aires, Argentina. Correo electrónico: solgrin@yahoo.com.ar

Para citar este artículo: Grinhauz, A. S., \& Castro, S. A. (2015). Un estudio exploratorio acerca de las fortalezas del carácter en niños argentinos. Avances en Psicología Latinoamericana, 33(1), 45-56. doi: dx.doi.org/10.12804/apl33.01.2015.04 
Keywords: Positive Psychology, strengths, children, emic

\section{Resumo}

Esta pesquisa centrou-se no segundo pilar da psicologia positiva -as virtudes e fortalezas do carácter- com o fim de explorar mediante um enfoque misto, é dizer, êmico -ético, as fortalezas auto percebidas em 163 crianças, $51 \%$ meninos e $49 \%$ meninas pertencentes à Cidade de Buenos Aires (Argentina). O 40\% dos participantes tinha 10 anos, o $28 \%$ tinha 11 anos e o $32 \%$ tinha 12 anos. Se pediu aos participantes que descreveram com suas palavras as fortalezas do carácter que reconheciam em si mesmos.

Dentro das fortalezas mais reportadas pelos participantes encontraram-se a integridade e a bondade. Dentro das menos reportadas como presentes acharam a espiritualidade, o amor pelo conhecimento e a prudência. Igualmente, encontraram-se diferenças nas fortalezas auto percebidas de acordo ao gênero e a idade. Nas respostas brindadas pelas crianças não surgiram fortalezas diferentes às previstas na classificação Values In Action Palavras-chave: Psicologia Positiva, Fortalezas, crianças, Êmico

A finales de los años noventa, Martin Seligman comenta que la psicología no comprende solamente el estudio de la enfermedad, la debilidad y el daño, sino también el estudio de los aspectos positivos del ser humano (Seligman \& Csíkszentmihályi, 2000). Este autor propuso reorientar la investigación científica hacia una mejor comprensión de los rasgos positivos que tienen los jóvenes y de todos aquellos aspectos que mejoren la calidad de vida de las personas (Fowler, Seligman \& Koocher, 1999; Linley, Joseph, Harrington \& Wood, 2006). En este sentido, la Psicología Positiva (PP) se define como el estudio científico de las experiencias positivas, los rasgos individuales positivos y las instituciones que facilitan su desarrollo (Gable \& Haidt, 2005; Seligman \& Csíkszentmihályi, 2000).
La PP abarca tres áreas principales: la subjetiva, la individual y la institucional. El área subjetiva estudia principalmente las experiencias subjetivas que son evaluadas positivamente, como las emociones positivas, la satisfacción con la vida y el bienestar. El área individual comprende los rasgos individuales positivos, tales como las fortalezas de carácter y las virtudes. El área institucional trata del estudio de los aspectos positivos de los grupos humanos en instituciones, que son el vehículo para que las personas se conviertan en mejores ciudadanos (Carr, 2007; Gable \& Haidt, 2005; Park \& Peterson, 2009; Seligman \& Csíkszentmihályi, 2000).

El estudio del segundo pilar de la PP —-fortalezas y virtudes de carácter- ocupa un papel central en la promoción del bienestar de las personas debido a que favorece el desarrollo de las otras dos áreas comentadas (Park, 2004a). El desarrollo de las fortalezas del carácter promueve la sensación subjetiva de bienestar y las emociones positivas, por un lado, y, por el otro, las instituciones positivas están formadas por personas de buen carácter (Park \& Peterson, 2009).

En la última década, Peterson y Seligman (2004) propusieron el estudio de los rasgos positivos basados en una rigurosa metodología científica. La clasificación de las fortalezas y virtudes era un paso importante y necesario para el estudio científico de la excelencia moral. Los autores desarrollaron una clasificación de las virtudes humanas que resultó la piedra angular de las investigaciones en ese campo y que, al mismo tiempo, sirvió como base para el desarrollo de las intervenciones psicológicas. Para el desarrollo de la clasificación, Peterson y Seligman (2004) consideraron las tradiciones filosóficas y religiosas más importantes de oriente y de occidente. A partir de esta revisión, los autores encontraron que eran seis las virtudes fundamentales que podían reconocerse en la mayoría de las tradiciones religiosas y filosóficas: el coraje, la justicia, la humanidad, la templanza, la sabiduría y la trascendencia (Dahlsgaard, Peterson $\&$ Seligman, 2005). Posteriormente, un grupo de 
académicos propuso una lista tentativa de las fortalezas humanas que podían incluirse a ese grupo de virtudes. Esta lista fue refinada por medio de una serie de debates y acuerdos, dando lugar a una clasificación de 24 fortalezas del carácter, que podían ubicarse en las seis virtudes comentadas. En la tabla 1 se presenta la clasificación de las seis virtudes y 24 fortalezas propuestas por Peterson y Seligman (2004).

Tabla 1

Clasificación de las seis virtudes y 24 fortalezas (Peterson \& Seligman, 2004)

\begin{tabular}{ll}
\hline \multicolumn{1}{c}{ Virtudes } & \multicolumn{1}{c}{ Fortalezas } \\
\hline Coraje & $\begin{array}{l}\text { Valentía, persistencia, integridad y } \\
\text { vitalidad }\end{array}$ \\
Justicia & Ciudadanía, liderazgo e imparcialidad \\
Humanidad & Amor, bondad e inteligencia social \\
Sabiduría & $\begin{array}{l}\text { Perspectiva, apertura mental, amor por } \\
\text { el saber, curiosidad y creatividad }\end{array}$ \\
Templanza & $\begin{array}{l}\text { Clemencia, humildad, prudencia y auto- } \\
\text { rregulación }\end{array}$ \\
Trascendencia & $\begin{array}{l}\text { Apreciación por la belleza, gratitud, } \\
\text { esperanza, humor y espiritualidad }\end{array}$ \\
\hline
\end{tabular}

La clasificación fue denominada Values in Action (VIA) y fue desarrollada con el objetivo de facilitar el lenguaje común para la investigación en PP. Los estudios que tomaron como base esta clasificación permitieron investigar la existencia de asociaciones entre las fortalezas y otros constructos psicológicos tales como el éxito escolar, la conducta prosocial y las competencias de los jóvenes (Park, 2004b). Las fortalezas también resultaron importantes predictoras de la prevención de problemas del comportamiento, tales como la agresividad, la adicción al consumo de sustancias y los trastornos depresivos en las poblaciones más jóvenes.

Según Park (2004b), las fortalezas pueden ser enseñadas y adquiridas con la práctica. En este sentido, la infancia constituye una de las etapas propicias para el desarrollo de estas (Richards \&
Huppert, 2011). Fortalezas tales como la autorregulación, la prudencia y la clemencia están relacionadas con un desarrollo psicológico positivo y una mejor adaptación en las escuelas (Cillessen \& Rose, 2005). Asimismo, la curiosidad y la apertura mental facilitan el aprendizaje y un mejor rendimiento académico (Kashdan \& Yuen, 2007). A su vez, el deseo de aprender presenta una relación positiva con la motivación intrínseca (Anderman \& Young, 1994), con el deseo de superación, con el interés en el propio desarrollo y con el valor del aprendizaje (Zimmerman, Bandura \& Martínez Pons, 1992). También, se encontraron relaciones significativas entre la gratitud y la satisfacción con la experiencia escolar (Froh, Sefick \& Emmons, 2008), y entre la valentía y la recuperación de enfermedades físicas y de trastornos psicológicos (Peterson, Park, \& Seligman, 2005). Además, la esperanza está asociada positivamente con la infancia, con la percepción de competencia y la autoestima (Marques, Pais Ribeiro \& Lopez, 2007). En relación con el humor, los niños que poseen esta fortaleza tienden a ser más asertivos y competentes en contextos sociales y académicos (Erickson \& Feldstein, 2007).

Según Peterson y Seligman (2004), esta clasificación era tentativa y podía ser modificada en función de los avances científicos en el campo. A pesar de que los autores intentaron evitar posibles sesgos culturales en su construcción, aún faltan estudios empíricos que confirmen la universalidad de la clasificación VIA (Diener, 2006). Algunas excepciones son los estudios desarrollados por Diener (2006) y por Park, Peterson y Seligman (2006) quienes, mediante la administración de cuestionarios on-line, confirmaron que algunos participantes de regiones diversas reconocían la mayoría de las fortalezas y virtudes contenidas en la clasificación VIA. Estos estudios abarcaron diferentes grupos culturales que iban desde Kenya hasta Groenlandia.

Sin embargo, cuando se le pide a un grupo de personas que se autodescriba en función de sus fortalezas del carácter, se enfrentan dos perspectivas 
muy distintas según la aproximación utilizada. Si se administra un cuestionario con un set de virtudes preestablecidas, las fortalezas del carácter pueden reducirse a un puñado de rasgos que están presentes en la mayoría de las personas en todas las sociedades (enfoque top-down). Por otro lado, algunas fortalezas del carácter pueden resultar más salientes en algunas sociedades que en otras, debido a razones históricas, culturales e idiosincráticas (enfoque bottom-up). Es decir, que en función del patrón de comparación que se utilice, las fortalezas pueden estar sobre o subrepresentadas en diferentes sociedades. En este sentido, las culturas asiáticas se caracterizan por otorgarle mayor importancia a los vínculos humanos, por lo tanto una de las fortalezas de este tipo de sociedades es la capacidad de trabajo en equipo. En contraste, las culturas más occidentales son reconocidas como más individualistas, predominando los intereses del individuo por sobre los del grupo. Por lo tanto, se supone que en función de estas variaciones culturales emergerá un patrón diferente de fortalezas del carácter (Park, Peterson \& Seligman, 2006).

Aunque se ha investigado en diferentes contextos culturales un conjunto muy variado de constructos derivados de la PP, el enfoque comúnmente utilizado ha sido el ético impuesto (Berry, 1969; Triandis, Malpass \& Davidson, 1972). Este enfoque top-down es el que predominó en la investigación en PP. La adopción de esta estrategia de trabajo hace que se desvanezcan las variantes émicas locales, quedando estas subrepresentadas. Esto lleva a que los constructos psicológicos estudiados revistan características pseudoéticas o que en suma sean "falsamente universales" (Castro Solano, 2012). Por lo tanto, para poder entender cuál aspecto de los constructos estudiados son realmente universales (éticos) y cuáles derivan de una variación local (émicos) resulta muy importante el estudio sistemático de las diferencias individuales, producto de los diferentes contextos culturales (Berry, Poortinga, Segall \& Dasen, 2002). Algunos autores consideran que el estudio de estas variaciones es un paso necesario para comprobar la universalidad de los principios psicológicos (Sinha, 1997).

En una revisión reciente de las investigaciones realizadas con constructos de la PP en América Latina (Castro Solano, 2012), se comentó que muchos psicólogos regionales eran propensos a aceptar la supuesta universalidad de estos constructos. Aunque muchos expertos destacaron la importancia de estudiar la variación sistemática de los constructos de la PP sobre la base de los factores culturales, la investigación transcultural y émica no es frecuente y, a excepción de algunos estudios relacionados con el bienestar (Alarcón, 2002; Albuquerque \& Trocoli Torres, 2004; Anguas Plata, 2001; Ballesteros, Medina \& Caycedo, 2006; Casullo \& Castro Solano, 2000), resulta escasa en contextos latinoamericanos. En particular, las fortalezas de carácter permanecen como un constructo sin explorar en los países de América del Sur, con la excepción de algunos estudios de Argentina dirigidos a población adulta (Cosentino, 2011; Cosentino \& Castro Solano, 2012) no encontrándose estudios en niños.

En función de lo comentado, el presente estudio se centra en el segundo pilar de la PP — las virtudes y fortalezas del carácter- con el fin de explorar las fortalezas que autoperciben los niños de la ciudad de Buenos Aires (Argentina) utilizando un enfoque mixto, es decir, émico-ético. A su vez, esta investigación tiene por objetivo verificar si existe un ajuste entre la clasificación VIA y la agrupación resultante de los rasgos positivos en esta población de América Latina. Finalmente, se busca determinar si existen diferencias entre las fortalezas de carácter autopercibidas de acuerdo con el sexo y la edad.

\section{Participantes y procedimiento}

Participó de este estudio una muestra de conveniencia compuesta por 163 niños que tenían entre diez y doce años y que asistían a escuelas públicas y privadas de la Ciudad de Buenos Aires (Argentina). 83 varones (51\%) y 79 mujeres (49\%). 
46 alumnos tenían 10 años (28\%), 42 alumnos tenían 11 años (26\%) y 74 alumnos tenían 12 años (46\%). La muestra se hallaba balanceada por sexo. E1 $98 \%$ de los padres trabajaban y en su mayoría eran empleados (66\%) o profesionales $(29 \%)$ y el $42 \%$ de los padres tenía el secundario completo. Por lo tanto, se trató de una muestra que, en su mayoría, pertenecía a la clase socioeconómica media. Se solicitó la colaboración voluntaria, anónima y no remunerada de los entrevistados.

A los participantes se les solicitó que respondieran una pregunta abierta, indicando en esta las cualidades o cosas buenas que reconocían en sí mismos y aquellas que sus familiares y amigos valoraban en ellos. Se les pidió que utilizaran sus propias palabras y que, en no más de una página, contestaran esta tarea. Dado el carácter relativamente abstracto de la tarea, se decidió ilustrar con ejemplos de qué se trataba la tarea requerida. Para ello previamente se leyó un relato donde se tomaron seis personajes prototípicos de la novela Harry Potter de J. K. Rowling. Cada uno de estos personajes representaba las seis virtudes del carácter planteadas por el modelo de Peterson y Seligman, (2004). Así, Harry Potter ejemplificaba el coraje, Hermione representaba la sabiduría; Ron, la humanidad; Albus Dumbledore, la templanza; Minerva McGonagall, la justicia y los padres de Harry Potter, la trascendencia. No se explicitaron las fortalezas para no inducir las respuestas de los evaluados. Una vez leído el relato, se repartieron las hojas en blanco con la consigna y se les dieron treinta minutos para responder a la tarea. La actividad tuvo una duración de sesenta minutos.

Asimismo, se solicitó información sobre el sexo, la edad, el nivel académico y ocupacional de los padres.

\section{Análisis de contenido}

Se realizó un análisis de contenido en el que se identificaron las fortalezas de carácter derivadas del relato llevado a cabo por cada niño de la muestra.
Dos jueces expertos codificaron las palabras (cualidades admiradas en sí mismo y por los otros), con el propósito de identificar si se trataba de alguna de las 24 fortalezas del carácter de la clasificación VIA. El punto de inicio fue identificar si cada palabra respondía a las fortalezas de la clasificación VIA o si se trataba de un sinónimo. Por ejemplo, para la fortaleza persistencia, se tomaron las palabras persistencia y algunas características que aludían a esta fortaleza, tales como esfuerzo, voluntad, perseverancia o constancia. Para la fortaleza bondad, se consideraron las palabras amistad, generosidad y amabilidad. La fortaleza de carácter integridad fue codificada como tal si el niño hacía mención a la lealtad, el respeto, la autenticidad o la honestidad. La fortaleza ciudadanía fue considerada si el niño hacía alusión a la cooperación con el grupo de pares, la escuela o la familia. Finalmente, ningún niño se ha descrito como con autocontrol, pero sí se han descrito como tranquilos o pacientes. En este caso se codificó la fortaleza autorregulación. Las palabras no fueron codificadas si se trataba de alguna característica negativa. Es decir, la imprudencia no fue codificada como valentía, la timidez no fue codificada como prudencia y las bromas de mal gusto como humor (Peterson \& Seligman, 2004). Tampoco se codificaron la inteligencia y la belleza, al no ser consideradas fortalezas de carácter. En la tabla 2 se presentan algunos ejemplos de las codificaciones realizadas para cada fortaleza registrada. Se registró un alto grado de acuerdo entre los dos jueces codificadores (kappa > .70).

\section{Resultados}

\section{Frecuencias de las fortalezas reconocidas}

Una vez realizada la codificación, se procedió a calcular las frecuencias de aparición de cada fortaleza que los niños encuestados habían identificado. Se obtuvieron 760 cualidades personales. $96(11.92 \%)$ respuestas resultaron inclasificables. En estos casos se trataba en su mayoría de casos 
Tabla 2

Ejemplos de fortalezas consideradas por niños de 10 a 12 años

\begin{tabular}{ll}
\hline $\begin{array}{c}\text { Fortalezas (Peterson } \\
\text { \& Seligman, 2004) }\end{array}$ & \multicolumn{1}{c}{ Ejemplos } \\
\hline Bondad & $\begin{array}{l}\text { Me considero un buen amigo que } \\
\text { se preocupa por sus compañeros. } \\
\text { Siempre digo la verdad. Tengo } \\
\text { respeto por mis maestros, padres y } \\
\text { compañeros. }\end{array}$ \\
Cuntegridad & $\begin{array}{l}\text { Cuando me saco una buena nota, } \\
\text { no soy de decirlo por ahí. }\end{array}$ \\
Cuando trabajo en grupo, me gusta \\
que todos podamos participar por \\
igual. \\
Digo a mis padres, amigos o her- \\
manos que los quiero. \\
Nomor
\end{tabular}

en los que no se había entendido la consigna. Por ejemplo, se citaban las frases "soy un buen alumno/a"; "tengo mucha personalidad" que no se pudieron ubicar en ninguna de las fortalezas de la clasificación VIA. La palabra inteligencia se citó en 69 respuestas $(8.57 \%)$. También se consignaron las palabras belleza o lindo/a en diez respuestas $(1.24 \%)$. En resumen, el total de palabras que se pudieron clasificar fue de 664 (88.08\%).

Cada niño autopercibió un promedio de cuatro fortalezas. Las fortalezas que resultaron más frecuentes fueron la integridad (lealtad, respeto, honestidad, autenticidad) perteneciente a la virtud coraje, seguida por la fortaleza bondad (generosidad, amabilidad) perteneciente a la virtud humanidad. Por su parte, las fortalezas menos nombradas fueron la gratitud, el esfuerzo o la persistencia y la inteligencia social.

Las fortalezas relacionadas con la espiritualidad, el amor por el conocimiento, la creatividad, la apertura mental y la apreciación se hallaban subrepresentadas ( $<1 \%$ de las menciones), y particularmente, no hubo menciones de las fortalezas perspectiva, curiosidad, prudencia y vitalidad.

En la tabla 3 se citan las fortalezas de carácter con mayor frecuencia de aparición. Se excluyeron del análisis las fortalezas consignadas que tenían una frecuencia menor al $1 \%$.

Tabla 3

Frecuencia de fortalezas autopercibidas

\begin{tabular}{llcc}
\hline \multicolumn{1}{c}{ Fortaleza } & \multicolumn{1}{c}{ Virtud } & Frecuencia & $\begin{array}{c}\text { Porcentaje } \\
(\%)\end{array}$ \\
\hline Integridad & Coraje & 128 & 78.5 \\
Bondad & Humanidad & 124 & 76.1 \\
Humor & Trascendencia & 115 & 70.6 \\
Humildad & Templanza & 85 & 52.1 \\
Imparcialidad & Justicia & 46 & 28.2 \\
Clemencia & Templanza & 39 & 23.9 \\
Amor & Humanidad & 38 & 23.3 \\
Valentía & Coraje & 35 & 21.5 \\
Ciudadanía & Justicia & 27 & 16.6 \\
Autocontrol & Templanza & 26 & 16 \\
Gratitud & Trascendencia & 15 & 9.2 \\
Persistencia & Coraje & 14 & 8.6 \\
Inteligencia & Sabiduría & 10 & 6.1 \\
Social & & & \\
\hline
\end{tabular}

\section{Agrupamiento de las Fortalezas}

Con el propósito de establecer el agrupamiento de las fortalezas, se llevó a cabo un análisis de correspondencias múltiples. Este análisis permite escalar las variables cualitativas y analizar las relaciones entre ellas. Es una alternativa cualitativa del análisis factorial exploratorio para variables discretas y permite detectar la relación entre las variables analizadas. Se incluyeron en el análisis solo aquellas fortalezas con alta frecuencia de aparición ( $>20 \%$ ). Como resultado, se obtuvieron dos dimensiones, la primera de ellas explicaba un 19\% de la inercia total.

En la primera dimensión se obtuvieron, en un extremo, las fortalezas relacionadas con la presencia de la integridad, la bondad, la ciudadanía y el amor; mientras que en el otro extremo estas 
fortalezas se hallaban ausentes. Estos resultados muestran que las fortalezas nombradas registraron la mayor capacidad de discriminación en la primera dimensión, lo cual indica que los niños reconocieron conjuntamente las fortalezas de las virtudes del coraje, la justicia y la humanidad como asociadas. En este sentido, los rasgos positivos de un niño serían entonces hacer lo que se predica que es correcto, ayudar a los demás sin esperar nada a cambio, expresar afecto por los demás y comprometerse con el grupo de pertenencia.

Por otro lado, la segunda dimensión explicó un $16 \%$ de la inercia total. Las fortalezas con mayor capacidad de discriminación fueron la humildad, la imparcialidad, la valentía y la clemencia. El énfasis en esta dimensión estuvo puesto en las fortalezas del carácter, pertenecientes a las virtudes de la templanza, de la justicia y del coraje. En este caso, un segundo perfil mostraría que los niños expresan fortalezas que tienen que ver con no ostentar con los propios logros, realizar juicios sociales equitativos y objetivos, hacer lo correcto aun corriendo riesgos y comportarse de forma benévola hacia una persona que no lo merece.

\section{Diferencias individuales según sexo y edad}

En último término se intentó identificar las diferencias individuales según sexo y edad. En relación con el sexo, se encontraron diferencias individuales solamente en la fortaleza amor a favor de las mujeres $\left(14.7 \%\right.$ contra $\left.8.6 \%, \chi^{2}=4.28, p<.05\right)$. En el resto de las fortalezas no se encontraron diferencias estadísticamente significativas entre los sexos.

En cuanto a la edad, se calculó el coeficiente de Kruskall Wallis (ver datos en tabla 4). Los resultados muestran que los niños de doce años autopercibieron en mayor medida las fortalezas respeto, gratitud, perseverancia, humor, bondad, inteligencia social, ciudadanía, autocontrol, capacidad de perdonar y valentía.

\section{Discusión}

Este estudio tuvo como principal objetivo explorar las fortalezas de carácter que autopercibieron los niños de 10 a 12 años de población local. Los resultados obtenidos muestran que los niños argentinos han podido reconocer en su carácter

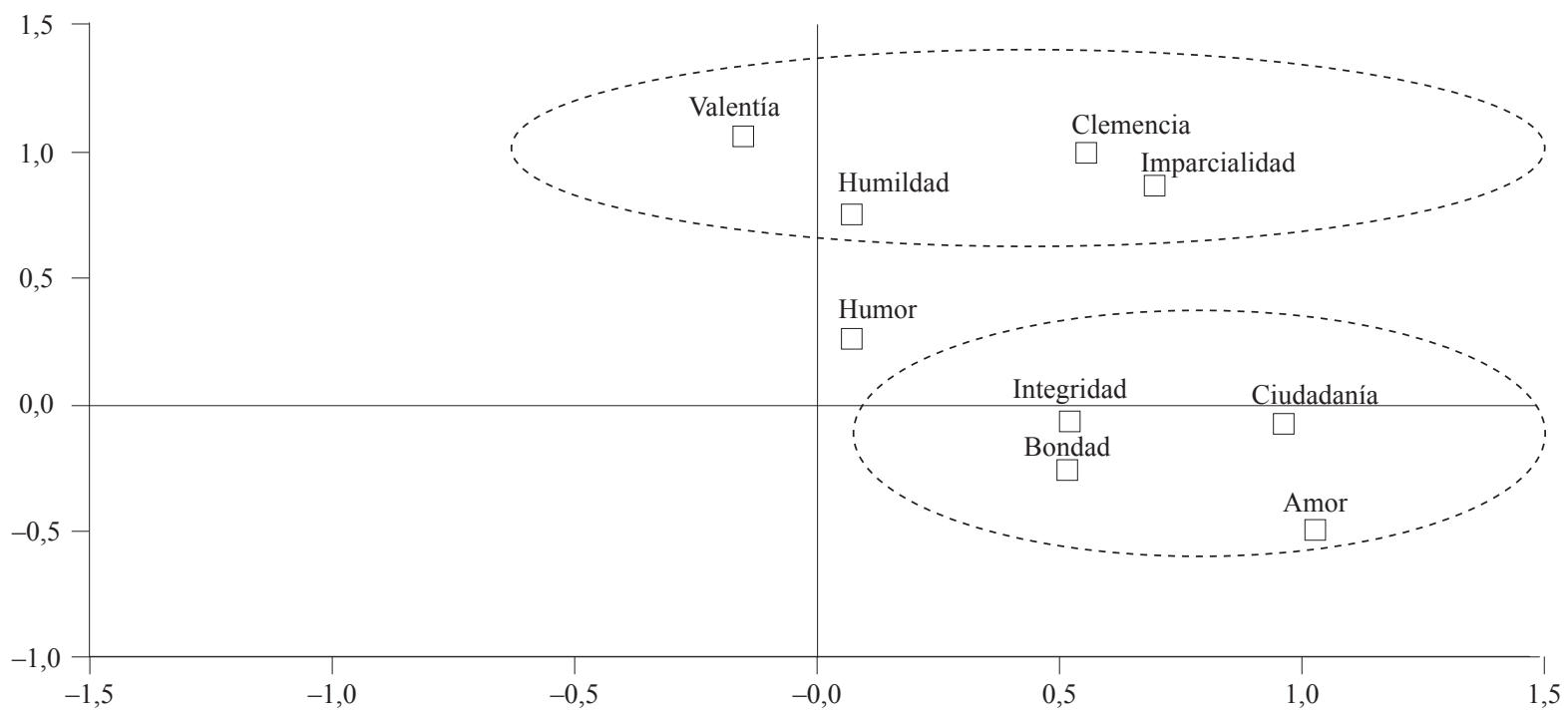

Figura 1. Análisis de correspondencias múltiples de fortalezas más frecuentes 
Tabla 4

Diferencias entre las fortalezas del carácter de acuerdo con la edad

\begin{tabular}{lrrrrr}
\hline \multicolumn{1}{c}{ Fortalezas } & $\begin{array}{c}\text { Kruskal } \\
\text { Wallis }\end{array}$ & $\begin{array}{c}\text { Sig. } \\
\text { (2Gl.) }\end{array}$ & \multicolumn{2}{c}{ Rango promedio } \\
\hline Respeto & & & 10 & 11 & 12 \\
Gratitud & 13.83 & $* *$ & 70.66 & 85.01 & 93.35 \\
Perseverancia & 22.24 & $* *$ & 74.50 & 77.01 & 94.49 \\
Humor & 19.64 & $* *$ & 76.25 & 76.81 & 93.45 \\
Bondad & 6.54 & $*$ & 71.40 & 82.68 & 90.16 \\
Inteligencia Social & 11.78 & $* *$ & 77.00 & 80.62 & 89.30 \\
Ciudadanía & 18.74 & $* *$ & 68.50 & 79.26 & 93.58 \\
Autocontrol & 18.53 & $* *$ & 70.81 & 76.69 & 94.08 \\
Clemencia & 10.97 & $*$ & 71.73 & 81.31 & 95.10 \\
Valentía & 21.39 & $* *$ & 64.50 & 81.42 & 94.59 \\
\hline
\end{tabular}

${ }^{*} p<.05$, bilateral. $* * p<.01$ bilateral.

trece de las 24 fortalezas propuestas por la clasificación VIA de Peterson y Seligman (2004). Las fortalezas que resultaron más frecuentes fueron la integridad, perteneciente a la virtud coraje; la bondad, perteneciente a la virtud humanidad; y el humor, perteneciente a la virtud trascendencia. Esto quiere decir que los niños argentinos se autopercibieron como personas fieles a sus valores, dispuestos a ayudar sin pedir nada a cambio, que gustan de hacer reír a los demás y de no ostentar con sus logros.

Estos resultados presentan coincidencias con estudios previos llevados a cabo con jóvenes norteamericanos, japoneses y españoles donde la bondad y el humor también resultaron ser las fortalezas más registradas (Giménez, 2010; Park \& Peterson, 2006; Shimai, Otake, Park, Peterson \& Seligman, 2006). Asimismo, si se comparan los resultados de la presente investigación con otro estudio llevado a cabo también en Argentina pero dirigido a población adulta (Cosentino \& Castro Solano, 2012) puede advertirse que la integridad y la bondad también resultaron ser dos de las fortalezas más nombradas por los participantes. Estos datos mostrarían que, en Argentina, las fortalezas que tienen que ver con la lealtad, la honestidad, el ser auténtico y el ayudar a los otros sin esperar nada a cambio cobran mucha relevancia tanto para niños como para adultos.

No obstante, Park y Peterson (2006) encontraron que, a diferencia de lo hallado en Argentina, la integridad fue la fortaleza menos registrada en niños norteamericanos de tres a nueve años. Esta diferencia puede deberse a que la cualidad de ser auténtico y fiel a los propios valores no suele expresarse en niños menores de diez años (Peterson \& Seligman, 2004) pero, una vez alcanzada esa edad, los niños comienzan a manifestar una mayor preocupación por las normas morales, los juegos comienzan a ser regulados y surge una necesidad imperiosa de respetar las reglas (Park \& Peterson, 2006; Piaget, 1932).

Por otra parte, las fortalezas menos nombradas o directamente no nombradas en el presente estudio fueron la espiritualidad, el amor por el conocimiento, la creatividad, la apertura mental, la apreciación de la belleza, la perspectiva, la curiosidad, la prudencia, el liderazgo y la vitalidad. De acuerdo con Park y Peterson (2006), se considera esperable que la mayoría de estas fortalezas no sean expresadas por niños de la edad de la muestra aquí analizada, debido a que requieren de un grado de madurez psicosocial más elevado que suele alcanzarse recién en la adolescencia. Estos resultados se encuentran en consonancia con los obtenidos por investigaciones previas provenientes de diferentes países, tales como Estados Unidos, España y Argentina (Cosentino \& Castro Solano, 2012; Giménez, 2010; Park \& Peterson, 2006; Peterson \& Park, 2003).

Por otro lado, el segundo objetivo de la presente investigación procuró verificar la existencia de un ajuste entre la clasificación VIA y la agrupación resultante de los rasgos positivos. Los resultados mostraron dos dimensiones. La primera dimensión estuvo integrada por las fortalezas integridad, bondad, ciudadanía y amor. De esta forma, los 
niños reconocieron conjuntamente las fortalezas pertenecientes a las virtudes del coraje, la justicia y la humanidad. En la segunda dimensión, por su parte, se agruparon la humildad, la imparcialidad, la valentía y la clemencia. En este caso, los niños reconocieron conjuntamente las fortalezas pertenecientes a las virtudes de la templanza, de la justicia y del coraje.

A partir de lo dicho anteriormente, puede decirse que la primera dimensión supone la necesidad de una interacción positiva con el otro, en la cual se preserven aquellos valores tan importantes para los niños, como pueden ser la honestidad, la lealtad, la responsabilidad y la amistad verdadera. Pueden advertirse muchos aspectos en común entre esta primera agrupación y la dimensión de autotrascendencia de Schwartz (2001) integrada por los dominios motivacionales de benevolencia y universalismo. En este sentido, la benevolencia centra su motivación en la preocupación por el bienestar del prójimo, por medio de la interacción diaria de los individuos, mientras que el universalismo es motivado por los valores del entendimiento, la apreciación, la tolerancia y la protección.

La segunda dimensión es de carácter más restrictivo, al hacer referencia al mantenimiento de cierto tipo de equilibrio, cohesión o armonía interna en pos de poder controlar los propios excesos para que la interacción y el funcionamiento del grupo se lleven armoniosamente. Esta agrupación tiene muchos aspectos en común con la dimensión de conservación que plantea Schwartz (2001), la cual remarca la importancia de la auto-restricción sumisa, la conservación de las prácticas tradicionales y la protección de la estabilidad social.

Finalmente, el tercer objetivo perseguido por el presente estudio consistió en analizar las diferencias en las fortalezas de carácter autopercibidas por los niños de acuerdo con el género y la edad. Los resultados mostraron que el amor fue la única fortaleza que diferenciaba entre varones y mujeres, siendo el género femenino quien más nombró esta fortaleza. Estos resultados se encuentran en con- sonancia con los hallados por Shimai et al. (2006) donde jóvenes japonesas y norteamericanas se reconocieron más predispuestas que los varones a dar y recibir muestras de afecto.

En relación con la edad, estudios previos muestran que a mayor edad se espera que exista una mayor presencia de fortalezas (Peterson \& Park, 2003; Peterson \& Seligman, 2004). Estos resultados coinciden con lo hallado en la presente investigación. Los niños de doce años reconocieron en mayor medida el respeto, la gratitud, la perseverancia, el humor, la bondad, la inteligencia social, la ciudadanía, el autocontrol, la capacidad de perdonar y la valentía como rasgos de su carácter, lo cual es esperable debido a que la mayoría de estas fortalezas requieren de un mayor desarrollo cognitivo y psicosocial para ser expresadas (Park \& Peterson, 2006).

En cuanto a las limitaciones de este estudio, puede mencionarse que, al ser de corte transversal, no se puede saber si las fortalezas autopercibidas por los niños de diez a doce años irán cambiando a medida que el niño crezca y se enfrente a otras etapas de la vida como pueden ser la adolescencia o la adultez. Estudios previos evidencian que de acuerdo con el momento evolutivo, se expresan más algunas fortalezas que otras (Giménez, 2010; Park \& Peterson, 2006). Si bien en, este caso, la integridad fue la fortaleza más registrada tanto por niños como adultos argentinos, hay otras fortalezas que se asocian a las capacidades cognitivas de la persona, con lo cual suelen aparecer recién en la adultez como es el caso de la perspectiva o la prudencia (Park \& Peterson, 2006). Por este motivo, se recomienda para futuras investigaciones el desarrollo de estudios longitudinales que permitan evaluar tanto la estabilidad como los cambios respecto de las fortalezas del carácter.

Otra de las limitaciones tiene que ver con las características de la muestra, la cual está conformada por alumnos de instituciones de clase económica media. Sería oportuno en futuras investigaciones contar con una muestra más heterogénea donde 
participen niños de instituciones educativas de clase alta o de clase baja.

Para concluir, este estudio permitió ver que la clasificación VIA propuesta por Peterson y Seligman (2004) ha podido representar más de la mitad de las fortalezas autopercibidas por los niños Argentinos. Estos resultados coinciden con estudios previos llevados a cabo por Diener (2006) y por Park, Peterson y Seligman (2006) quienes confirmaron que participantes de regiones diversas reconocían la mayoría de las fortalezas contenidas en la clasificación VIA. Otro punto importante a destacar es que no surgieron fortalezas nuevas, es decir, los niños no mencionaron cualidades diferentes a las ya existentes en la clasificación VIA. Asimismo, el hecho de que muchas de las fortalezas pertenecientes a la clasificación VIA no fueran nombradas por los participantes de población local evidenciaría que, de acuerdo con la cultura de origen, hay fortalezas que se expresan más que otras. Si bien estudios previos han reconocido a las culturas occidentales como más individualistas, en este caso se pudo constatar todo lo contrario. De esta manera, respetar, ayudar y hacer reír al prójimo constituyen las fortalezas más autopercibidas por los niños argentinos en su carácter.

\section{Referencias}

Alarcón, R. (2002). Estudios sobre psicología latinoamericana. Lima: Universidad Ricardo Palma.

Albuquerque, A. S., \& Tróccoli, B. T. (2004). Desenvolvimento de uma escala de bemestar subjetivo. Psicologia: Teoria e Pesquisa, 20, 153-164.

Anderman, E. M., \& Young, A. J. (1994). Motivation and strategy use in science: individual differences and classroom effects. Journal of Research in Science Teaching, 31, 811-831.

Anguas Plata, A. M. (2001). Identificación y validación del significado de bienestar subjetivo en México: Fundamentos para el desarrollo de un instrumento de medición. Interamerican Journal of Psychology, 35, 163- 183.
Ballesteros, B., Medina, A., \& Caycedo, C. (2006). El bienestar psicológico definido por asistentes a un servicio de consulta psicológica en Bogotá, Colombia. Universitas Psychologica, 5(2), 239-258.

Berry, J. W. (1969). On cross-cultural comparability. International Journal of Psychology, 4(2), 119128.

Berry, J. W., Poortinga, Y. H., Segall, M. H., \& Dasen, P. R. (2002). Cross-cultural psychology: research and applications. ( $2^{\text {nd }}$ Ed.). New York: Cambridge University Press.

Carr, A. (2007). Psicología positiva: La ciencia de la felicidad. Barcelona: Paidós.

Castro, S. A. (2012). La Psicología Positiva en América Latina. Desarrollos y perspectiva. Psiencia Latin American Journal of Psychological Science, 4(2), 108-116.

Casullo, M. M., \& Castro Solano, A. (2000). Evaluación del bienestar psicológico en estudiantes adolescentes argentinos. Revista de Psicología de la Pontificia Universidad Católica de Perú, 18(2), 36-68.

Cillessen, A., \& Rose, A. (2005). Understanding popularity in the peer system. Current Directions in Psychological Science, 14: 102-105.

Cosentino, A. C. (2011). Fortalezas del carácter en militares argentinos (Tesis Doctoral Universidad de Palermo, Argentina).

Cosentino, A. C., \& Castro Solano, A. (2012). Character Strengths: A Study of Argentinean Soldiers. The Spanish Journal of Psychology, 15(1), 199-215.

Dahlsgaard, K., Peterson, C., \& Seligman, M. (2005). Shared virtue: The convergence of valued human strengths across culture and history. Review of General Psychology, 9, 203-213.

Diener, E. (2006). Guidelinesfor national indicators of subjective well-being and ill-being. Applied Research in Quality of Life, 1, 151-157.

Erickson, S. J., \& Feldstein, S. W. (2007). Adolescent Humor and its relationship Coing, Defense Strategies, Psychological Distress, and Well Being. 
Child Psychiatry and Human Development, 37, 255 - 271.

Fowler, R. D., Seligman, M. P., \& Koocher, G. P. (1999). The APA 1998 Annual Report. American Psychologist, 54, 537-568.

Froh, J., Sefick, W., \& Emmons, R. (2008). Counting blessings in early adolescents. An experimental study of gratitude and subjective well-being. Journal of School Psychology, 46(2), 213233.

Gable, S. L., \& Haidt, J. (2005). What (and why) is positive psychology? Review of General Psychology, 9, 103-110.

Giménez Hernández, M. (2010). La medida de las fortalezas psicológicas en adolescentes VIA youth: Relación con clima familiar, psicopatología y bienestar psicológico. (Tesis doctoral, Universidad Complutense de Madrid, Madrid, España).

Kashdan, T. B., \& Yuen, M. (2007). Wether highly curious students thrive academically depends on perceptions about the school learning environment: a study of Hong Kong adolescents. Motivation and Emotion, 31, 260-270.

Linley, A., Joseph, A., Harrington, S., \& Wood, A. (2006). Positive psychology: Past, present and (possible) future. The Journal of Positive Psychology, 1(1), 3-16.

Marques, S. S., Pais-Ribeiro, J. L., \& Lopez, S. J. (2007). Hope in relation to life satisfaction, mental-health, and self-worth in students. Póster presentado en el X Congreso Europeo de Psicología, Praga, República Checa.

Park, N. (2004a). The role of subjective well-being in positive youth development. The Annals of the American Academy of Political and Social Science, 591, 25-39.

Park, N. (2004b). Character strengths and positive youth development. The Annals of the American Academy of Political and Social Science, 591, 40-54.

Park, N., \& Peterson, C. (2006). Character Strengths and happiness among young children: Content analysis of parental descriptions. Journal of Happiness Studies, 7, 323-341.

Park, N., \& Peterson, C. (2009). Achieving and sustaining a good life. Perspective son Psychological Science, 4, 422-428.

Park, N., Peterson, C., \& Seligman, M. E. P. (2006). Character strengths in fifty-four nations and the fifty US states. Journal of Positive Psychology, $1,118-129$.

Peterson, C., \& Park, N. (2003). Positive psychology as the evenhanded positive psychologist views it. Psychological Inquiry, 14, 141-146.

Peterson, C., \& Seligman, M. E. P. (2004). Character strengths and virtues: A classification and handbook. Washington, DC: American Psychological Association-New York: Oxford University Press.

Peterson, C., Park, N., \& Seligman, M. E. P. (2005). Orientations to happiness and life satisfaction: The full life versus the empty life. Journal of Happiness Studies, 6(1), 25-41.

Piaget, J. (1932). The moral judgment of the child. New York: Harcourt, Brace, and World. (Translated by M. Worden).

Richards, M., \& Huppert, F. A. (2011). Do positive children become positive adults? Evidence from a longitudinal birth cohort study. Journal of Positive Psychology, 6, 75-87.

Seligman, M., \& Csíkszentmihályi, M. (2000). Positive Psychology: An introduction. American Psychologist 55(1), 5-14.

Schwartz, S. (2001). ¿Existen aspectos universales en la estructura y contenido de los valores humanos? En: M. Ros y V. Gouveia (Eds.), Psicología Social de los Valores Humanos (pp. 53-76). Madrid: Biblioteca Nueva.

Shimai, S., Otake, K., Park, N., Peterson, C., \& Seligman, M. E. P. (2006). Convergence of character strengths in American and Japanese Young Adults. Journal of Happiness Studies, 7, 311-322.

Sinha, D. (1997). Indigenizing psychology. In J. W. Berry, Y. H. Poortinga, \& J. Pandey (Eds.), 
Handbook of cross-cultural psychology: Vol. 1. Theory and method (2nd Ed.). Boston: Allyn \& Bacon.

Triandis, H., Malpass, R., \& Davidson, A. (1973). Psychology and Culture. Annual Review of Psychology, 24, 355-78.
Zimmerman, B. J., Bandura, A., \& Martinez-Pons, M. (1992). Self-motivation for academic attainment: The role of self-efficacy beliefs and personal goal setting. American Educational Research Journal, 29, 663-676. 